\title{
Radical Cation-Nucleophile Combination Reactions. The Effect of Structure of Nitrogen-Centered Nucleophiles on Reaction Rates
}

\author{
Vernon D. Parker* and Bjørn Reitstøen \\ Department of Chemistry and Biochemistry, Utah State University, Logan, Utah 84322-0300, USA
}

Parker, V. D. and Reitstøen, B., 1997. Radical Cation-Nucleophile Combination Reactions. The Effect of Structure of Nitrogen-Centered Nucleophiles on Reaction Rates. - Acta Chem. Scand. 51: 1035-1040. (C) Acta Chemica Scandinavica 1997.

The kinetics of the reactions of 9-phenylanthracene (PAH) radical cation with a number of nitrogen-centered nucleophiles related to pyridine have been studied in acetonitrile and dichloromethane containing $\mathrm{Bu}_{4} \mathrm{NPF}_{6}$. Relative rate constants with pyridine as the nucleophile in the standard reaction, were compared with those expected from the configuration mixing (CM) model assuming that the reactive center of the radical cation is either cationic (i) or radical (ii). There is no apparent positive correlation between observed reaction barriers and those

(i) $\mathrm{ArH}^{+}+\mathrm{N}: \rightarrow \mathrm{ArHN}^{+}$

(ii) $\mathrm{ArH}^{+}+\mathrm{N}: \rightarrow{ }^{+} \mathrm{ArHN}$

expected by either CM model treatment. Reaction (i) is considered to be 'forbidden' while reaction (ii) is 'allowed' by the CM model treatment. The initial gaps for reactions (ii) are estimated to be of the order of $30-40 \mathrm{kcal} \mathrm{mol}^{-1}$ lower than those for reactions (i). It is concluded that the reactions take place by pathway (i), in spite of the greater energy gap, but that the two pathways would be experimentally indistinguishable since ${ }^{+} \mathrm{ArHN}^{-}$is expected to be unstable with respect to $\mathrm{ArHN}^{+}$.

The reactions of 9-phenylanthracene $(\mathrm{PAH})$ radical cations with pyridine derivatives in acetonitrile and dichloromethane as solvents containing $\mathrm{Bu}_{4} \mathrm{NPF}_{6}$ have been observed to be rapid, low activation energy processes and were concluded to involve the direct attack of the nitrogen-centered nucleophile on the radical cation. ${ }^{1}$ This conclusion was contrary to the predictions based on the configuration mixing (CM) model made by Pross to explain the apparent low reactivities of some radical cations toward nucleophilic attack. ${ }^{2}$ The product of reaction (1) is doubly excited with respect to reactants and thus, in CM model terms, the process is 'forbidden'. ${ }^{3}$

$\mathrm{ArH}^{+}+\mathrm{N}: \rightarrow^{\cdot} \mathrm{ArHN}^{+}$

The CM model approach to this problem was later amplified. ${ }^{3}$ Subsequently, it has been shown that a number of radical cations react very rapidly with anionic nucleophiles ${ }^{4}$ and in some cases radical cations do not appear to be low in reactivity when compared with related carbocations. ${ }^{5}$

PAH radical cation (1) is persistent in acetonitrile and dichloromethane in the absence of nucleophiles and has

\footnotetext{
* To whom correspondence should be addressed.
}

been used extensively in mechanism studies. ${ }^{5-7}$ These studies have shown that substitution of the 10-hydrogen<smiles></smiles>

for deuterium (PAD) gives rise to an inverse kinetic isotope effect, $k_{\mathrm{H}} / k_{\mathrm{D}}$, of the order of $0.7-0.8$ during reactions of $\mathrm{PAH}^{+}$(or $\mathrm{PAD}^{+}$) with nucleophiles. The inverse deuterium kinetic isotope effect is a consequence of the $\mathrm{sp}^{2}$ hybridized carbon at position 10 undergoing hybridization change to $\mathrm{sp}^{3}$ in the transition state. ${ }^{8}$ The kinetic isotope effect data, along with a consideration of the energetics of reactions (2), ${ }^{7}$ clearly rule out an electron transfer mechanism as an alternative to the direct attack of the nucleophile on the radical cation. All available evidence points to a direct reaction between 
radical cation and nucleophile as the rate determined step in the combination process.

$\mathrm{ArH}^{+}+\mathrm{N}: \rightleftarrows \mathrm{ArH}+\mathrm{N}^{\cdot+}$

In this paper we examine the effect of wide variations in the structure of nitrogen-centered nucleophiles on the rates of the combination reaction with $\mathrm{PAH}^{+}$in acetonitrile and dichloromethane in order to determine whether or not an alternative formulation of the CM model better describes radical cation-nucleophile combination.

\section{Results and discussion}

The essential feature of the CM model is that transition state energies of most reactions can be predicted by considering only two major valence-bond contributors, 2 and 3. In the event that both the donor (D) and the acceptor (A) are closed-shell molecules or ions, all that

$$
\begin{array}{cccc}
\mathrm{D}: \mathrm{A} & { }^{+} \mathrm{D} \cdot \mathrm{A}^{-} & { }^{+} \mathrm{N} \cdot{ }^{3 *} \mathrm{ArH} \cdot & { }^{3 * \cdot} \mathrm{N} \cdot \mathrm{ArH}^{+} \\
\mathbf{2} & \mathbf{3} & \mathbf{4} & \mathbf{5}
\end{array}
$$

is necessary to convert $\mathbf{2}$ into $\mathbf{3}$ is a single electron shift (SES). Since 3 is a product configuration the reaction is expected to occur in a single step and the reaction is considered to be 'allowed'. When $\mathrm{A}$ is a radical cation $\left(\mathrm{ArH}^{+}\right)$combining with a nucleophile as in reaction (3), 3 is not a product configuration, and singlet-triplet

$\mathrm{ArH}^{+}+\mathrm{N}: \rightarrow^{\cdot} \mathrm{ArH}-\mathrm{N}^{+}$mechanism 1

excitation of $\mathrm{ArH}$ is required to achieve 4, a configuration common to both reactants and product. Thus, 4 is doubly excited relative to $\mathbf{2}$ and the radical cation-nucleophile combination reaction is considered to be 'forbidden'.

However, an alternative singly excited configuration, $5\left({ }^{3 *} \mathrm{~N}\right.$ : is the triplet state of the nucleophile), common to both reactants and initial products of the combination reaction, exists. This alternative is illustrated by eqn. (4), in which the product, ${ }^{+} \mathrm{ArH}-\mathrm{N}^{*}$, is singly excited with

$\mathrm{ArH}^{+}+\mathrm{N}: \rightarrow^{+} \mathrm{ArHN}^{\cdot}$ mechanism 2

${ }^{+} \mathrm{ArHN}{ }^{\cdot} \rightarrow{ }^{\circ} \mathrm{ArHN}^{+}$

respect to the reactant complex. This product is expected to be unstable with respect to $\mathrm{ArH}-\mathrm{N}^{+}$and conversion (5) need not be considered as a distinct step. In the terminology of the CM model, the initial energy gap (IG) for reaction (4) is simply the singlet-triplet excitation energy of the nucleophile $\mathrm{N}$. The initial gap refers to the energy difference between ground state reactant configuration and the excited state configuration common to both reactants and products. For nitrogencentered nucleophiles such as pyridine this excitation corresponds to $\Delta E_{\mathrm{ST}}\left(\mathrm{n} \rightarrow \pi^{*}\right)$. The attractive feature of this alternative mechanism is that the IG for the reactions can be considerably smaller than those corresponding to reaction (1).

In order to investigate the effect of nucleophile structure on the energetics of radical cation-nucleophile com- bination reactions, a series of aromatic compounds related to pyridine were selected as nucleophiles. The nucleophilic center in this series of compounds is the nitrogen lone-pair. The structures of the nitrogencentered nucleophiles, arranged in order of decreasing reactivity toward radical cations, are shown in Table 1. The radical cation of 9-phenylanthracene was selected as the other reactant. The reactive site in $\mathrm{PAH}^{+}$is the unhindered 10-position. The advantage of this radical cation is that $\mathrm{PAH}^{+}$is relatively long-lived in acetonitrile and dichloromethane, eliminating the complicating features of side reactions, while at the same time is unhindered toward nucleophilic attack at the 10-position. In the following paragraphs we first present our experimental results and then attempt to relate trends in our data to the alternative mechanisms 1 and 2.

Electrode potentials, ionization potentials, and singlettriplet excitation energies. Linear sweep voltammetry peak potentials for the oxidation of the nitrogen-centered nucleophiles in acetonitrile- $\mathrm{Bu}_{4} \mathrm{NPF}_{6}(0.1 \mathrm{M})$ are summarized in column 2 of Table 2 . The characteristic feature of these oxidation processes is that the initial charge transfers are accompanied by rapid chemical follow-up reactions. The peak potentials shown are therefore minimum values of the corresponding reversible peak potentials reflecting contributions from the kinetics of the chemical follow-up reactions. The differences in peak potentials for the nitrogen-centered nucleophiles as compared with that for the oxidation of PAH $\left(\Delta E^{\mathrm{p}}\right)$ are recorded in column 3 of Table 2 . Here again, the $\Delta E^{\mathrm{p}}$ can be considered to be minimum estimates of the reversible potential differences.

Gas phase ionization potentials are expected to be related to oxidation potentials in solution and the quantities are usually found to correlate linearly. Thus, the relative $E^{\mathrm{p}}$ can be expected to be a reasonably good measure of values of the relative vertical ionization potentials $[\mathrm{IP}(\mathrm{v})]$ of the nitrogen-centered nucleophiles. The relative values in parentheses tabulated in the third column of Table 2 were estimated from the relative $E^{\mathbf{p}}$ and adjusted to the literature value ${ }^{9}$ for pyridine $(9.58 \mathrm{eV})$. IP(v) values for compounds $\mathbf{1}$ and 5 have also been reported, ${ }^{9}$ and these deviate from our estimated values by $\approx 0.1 \mathrm{eV}$.

Singlet-triplet excitation energies $\left(\Delta E_{\mathrm{ST}} \mathrm{n} \rightarrow \pi^{*}\right)^{10}$ of three of the nitrogen-centered nucleophiles are known. These were observed to correlate well $\left(r^{2}=0.995\right)$ with the observed oxidation potentials ( $E^{\mathrm{p}}$ in column 1$)$. The other $\Delta E_{\mathrm{ST}} \mathrm{n} \rightarrow \pi^{*}$ listed in Table 2 were estimated from the correlation. Again, the estimated values are shown in parentheses.

Rate constants for radical cation-nucleophile reactions. Second-order rate constants for the reactions of $\mathrm{PAH}^{+}$with the nitrogen-centered nucleophiles in acetonitrile- $\mathrm{Bu}_{4} \mathrm{NPF}_{6}(0.1 \mathrm{M})$ and in dichloromethane$\mathrm{Bu}_{4} \mathrm{NPF}_{6}(0.2 \mathrm{M})$ at $298 \mathrm{~K}$ are summarized in Table 3. 
Table 1. Structures, numbers and names of nitrogen-centered nucleophiles.

Number

Table 2. Peak potentials for oxidation of nitrogen-centered nucleophiles in acetonitrile, ionization potentials and singlet-triplet excitation energies.

\begin{tabular}{lllll}
\hline Compound & $E^{\mathrm{pa}}$ & $\Delta E^{\mathrm{pb}}$ & $\mathrm{IP}(\mathrm{V}) / \mathrm{eV}^{c}$ & $\Delta E_{\mathrm{ST}}\left(\mathrm{n} \rightarrow \pi^{*}\right)^{d}$ \\
\hline $\mathbf{1}$ & 2.16 & 1.31 & $9.15(9.3)$ & $(75)$ \\
$\mathbf{2}$ & 1.67 & 0.82 & $(8.8)$ & $(66)$ \\
$\mathbf{3}$ & 2.46 & 1.61 & 9.58 & $(81)$ \\
$\mathbf{4}$ & 1.83 & 0.98 & $(9.0)$ & 68.6 \\
$\mathbf{5}$ & 1.96 & 1.11 & $9.19(9.1)$ & $(71)$ \\
$\mathbf{6}$ & 1.71 & 0.86 & $(8.8)$ & $(66)$ \\
$\mathbf{7}$ & 1.83 & 0.98 & $(9.0)$ & $(69)$ \\
$\mathbf{8}$ & 2.46 & 1.61 & $(9.6)$ & 81.2 \\
$\mathbf{9}$ & 2.23 & 1.38 & $(9.4)$ & 75.6 \\
$\mathbf{1 0}$ & 1.57 & 0.72 & $(8.7)$ & $(64)$ \\
$\mathbf{1 1}$ & 1.38 & 0.53 & $(8.5)$ & $(60)$ \\
\hline
\end{tabular}

${ }^{a}$ Peak potential for the oxidation of the nitrogen-centered nucleophiles in $\mathrm{CH}_{3} \mathrm{CN}-\mathrm{Bu}_{4} \mathrm{NPF}_{6}(0.1 \mathrm{M})$ vs. ferrocene ${ }^{+} /$ ferrocene at $298 \mathrm{~K}$. All oxidation processes were irreversible. ${ }^{b}$ The difference in peak potential for the oxidation of the nitrogen-centered nucleophiles in $\mathrm{CH}_{3} \mathrm{CN}-\mathrm{Bu}_{4} \mathrm{NPF}_{6}(0.1 \mathrm{M})$ and that for the $\mathrm{PAH}^{+} / \mathrm{PAH}$ couple at $298 \mathrm{~K}$. ${ }^{\circ}$ Vertical ionization potentials in $\mathrm{eV}$. Those in parentheses estimated from the relative $E^{\mathfrak{p}}$ values using the pyridine value as standard. ${ }^{d}$ Singlet-triplet energies in $\mathrm{kcal} \mathrm{mol}^{-1}$. Those in parentheses estimated from correlation vs. $E^{\mathfrak{p}}$.

Although there are small differences in the rate constants in the two solvents, the trends with structure are nearly the same, indicating little or no solvent effect on the rates of the reactions. The same conclusion has been
Table 3. Second-order rate constants for the reactions of nitrogen-centered nucleophiles with 9-phenylanthracene radical cation.

\begin{tabular}{lll}
\hline Compound & $\log k(\mathrm{AN})^{a}$ & $\log k(\mathrm{DCM})^{b}$ \\
\hline $\mathbf{1}$ & 8.45 & 8.72 \\
2 & 7.79 & 7.88 \\
3 & 7.08 & 6.90 \\
$\mathbf{4}$ & 7.04 & 6.59 \\
5 & 6.18 & 6.08 \\
6 & 5.81 & 5.58 \\
7 & 5.63 & 5.08 \\
$\mathbf{8}$ & 5.51 & 4.61 \\
9 & 5.26 & 4.04 \\
10 & 3.32 & 3.28 \\
11 & 2.32 & 1.95 \\
\hline
\end{tabular}

${ }^{a}$ Measured in acetonitrile- $\mathrm{Bu}_{4} \mathrm{NPF}_{6}(0.1 \mathrm{M})$ at $298 \mathrm{~K}$. ${ }^{b}$ Measured in dichloromethane-Bu $\mathrm{NPF}_{6}(0.2 \mathrm{M})$ at $298 \mathrm{~K}$.

drawn for similar studies of the reactions of radical cations with nitrogen-centered and other nucleophiles. ${ }^{1,4,11}$

Free energies of activation and initial gaps. The expectation $^{2,3}$ is that there will be a direct relationship between the free energies of activation and the IG in a reaction series. The IG for mechanism 1 [eqn. (6)] and mechanism 2 [eqn. (7)] indicate that the relative IG for the

$$
(\mathrm{IG})_{1}=I_{\mathrm{N}:}^{*}(\mathrm{~s})-\mathrm{A}_{\mathrm{E}}^{*}(\mathrm{~s})+\Delta E_{\mathrm{ST}}\left(\pi \rightarrow \pi^{*}\right)
$$$$
(\mathrm{IG})_{2}=\Delta E_{\mathrm{ST}}\left(\mathrm{n} \rightarrow \pi^{*}\right)
$$ 
reaction series between $\mathrm{PAH}^{+}$and the nitrogen-centered nucleophiles should simply be the difference in solution ionization potentials of $\mathrm{N}\left[I_{\mathrm{N}}^{*}(\mathrm{~s})\right]$ for mechanism 1 or the difference in singlet-triplet energies $\left[\Delta E_{\mathrm{ST}}\left(\mathrm{n} \rightarrow \pi^{*}\right)\right]$ of $\mathrm{N}$ for mechanism 2 . The solution ionization potentials are readily estimated from the gas phase values. There is an additional term in each of these equations, $R T \ln K_{\mathrm{ST}}^{*} / K_{\mathrm{DA}}^{\circ}$, involving the ratios of equilibrium constants for formation of the association complex in the ground and excited states. ${ }^{11}$ Since it is not possible to evaluate these terms, it is necessary to assume that they will not vary appreciably in this reaction series and will effectively cancel. $(\mathrm{IG})_{1}$ for the standard reaction, $\mathrm{PAH}^{+}+$pyridine, has been estimated to equal $112 \mathrm{kcal} \mathrm{mol}^{-1}$, which is somewhat higher than $\Delta E_{\mathrm{ST}}\left(\mathrm{n} \rightarrow \pi^{*}\right),(\mathrm{IG})_{2}$ for this reactant combination, which we estimate to be equal to $81 \mathrm{kcal} \mathrm{mol}^{-1}$. From the relative values of $\operatorname{IP}(\mathrm{v})$ and $\Delta E_{\mathrm{ST}}\left(\mathrm{n} \rightarrow \pi^{*}\right)$ for this series of reactions it appears that (IG) $)_{1}$ exceeds $(\mathrm{IG})_{2}$ by about $30-40 \mathrm{kcal} \mathrm{mol}^{-1}$ with the greatest difference being when the nucleophile is pyridine. No attempts were made to refine this estimate.

Since $I_{\mathrm{N}}^{*}(\mathrm{~s})$ is directly related to $E^{\mathrm{p}}$ and there appears to be a linear correlation between $\Delta E_{\mathrm{ST}}\left(\mathrm{n} \rightarrow \pi^{*}\right)$ and $E^{\mathrm{p}}$, the initial gaps for both mechanisms are expected to be proportional to $E^{\mathrm{p}}$. Linear relationships between the free energies of activation for the reaction series and the initial gaps would then require inverse linear relationships between $\log k$ and $E^{\mathrm{p}}$. Attempted linear correlation of $\log k$ vs. $E^{\mathbf{p}}$ failed to yield a reliable correlation line. Removal of the data from four of the nucleophiles $(2,3$, $\mathbf{8}$, and 9) resulted in a rough correlation with slope of $3.4\left(r^{2}=0.9\right)$, which indicates that the rate constants increase with this series of nucleophiles as $E^{p}$ increases, i.e., in the opposite sense than predicted by either eqn. (6) or $(7)$.

It is of interest to make comparisons of pairs of nitrogen-centered nucleophiles having the same ring arrangement but in which one or more of the rings are non-aromatic. Steric effects caused by ring junctures adjacent to the nitrogen center are expected to cancel in the comparisons of the pairs of nucleophiles. There are three such possible comparisons and these are summarized in Table 4. For all three comparisons, a negative $\Delta E^{\mathrm{p}}(\mathrm{B}-\mathrm{A})$, where $\mathrm{B}$ and $\mathrm{A}$ refer to the nucleophiles in

Table 4. A comparison of relative second-order rate constants and relative oxidation potentials for closely related nucleophile pairs.

\begin{tabular}{rccc}
\hline Nucleophile A & Nucleophile B & $\Delta E^{\mathrm{P}}(\mathrm{B}-\mathrm{A}) / V^{\mathrm{a}}$ & $\log k_{\mathrm{B}} / k_{\mathrm{A}}{ }^{b}$ \\
\hline $\mathbf{1}$ & $\mathbf{2}$ & -0.48 & -0.66 \\
$\mathbf{5}$ & 7 & -0.13 & -0.55 \\
$\mathbf{1 0}$ & $\mathbf{1 1}$ & -0.19 & -1.00 \\
\hline
\end{tabular}

${ }^{a}$ The difference in peak potentials for the oxidation of nucleophile $\mathrm{B}$ and $\mathrm{A}$. ${ }^{b}$ The rate constants $k_{\mathrm{A}}$ and $k_{\mathrm{B}}$ refer to the reactions between $\mathrm{PAH}^{+}$and nucleophiles $A$ and $B$ in acetonitrile. the pair, is associated with a negative $\log k_{\mathrm{B}} / k_{\mathrm{A}}$. The comparisons indicate that an increase in either $(\mathrm{IG})_{1}$ or $(\mathrm{IG})_{2}$ is accompanied by a corresponding decrease in the corresponding free energy of activation, i.e., the reaction becomes faster as the initial gap increases.

The nucleophile-pair data comparison described in the previous paragraph, as well as the more general rough correlation, indicates that there is not only a lack of positive correlation between initial gaps for both mechanisms and the observed free energies of activation but there appears to be at least a weak negative correlation between these quantities.

Inverse secondary deuterium kinetic isotope effects. In order to be assured that there is no change in mechanism as the structure of the nitrogen-centered nucleophile is changed, deuterium kinetic isotope effects were obtained from rate measurements using $\mathrm{PAH}^{+}$and $\mathrm{PAD}^{+}$(the deuterium is at the 10-position). The results are summarized in Table 5. Inverse secondary deuterium kinetic isotope effects were observed in all reactions with $k_{\mathbf{H}} / k_{\mathrm{D}}$ varying from 0.7 to 0.95 .

It is interesting to note that the reaction pair for which the electron transfer mechanism is most favorable $\mathrm{PAH}^{+}$/acridine (11) does not go by that mechanism as is evident from the observation of the inverse secondary deuterium kinetic isotope effect. Once again, the kinetic isotope effect is due to the change in hybridization of the $\mathrm{C}-10$ carbon from $\mathrm{sp}^{2}$ to $\mathrm{sp}^{3}$ upon attack by the nitrogen-centered nucleophile. Not only is the mechanism ruled out by the kinetic isotope effect, but the observed trend in rate constants is also inconsistent with an electron transfer mechanism (2).

An analogy to the alternative radical cation-nucleophile initial reaction steps. Nucleophilic attack on a radical cation does not necessarily involve a heteroatom as the attacking center. Radical cation-nucleophile combination reactions have been studied using anisole and phenol as nucleophiles. ${ }^{10,12-14}$ Thianthrene radical cation ${ }^{12-14}$ and $\mathrm{PAH}^{+15}$ react with both of these nucleophiles

Table 5. Kinetic isotope effects for the reactions of $\mathrm{PAH}^{+}$ and $\mathrm{PAD}^{+}+$with nitrogen-centered nucleophiles in acetonitrile and dichloromethane.

\begin{tabular}{lll}
\hline Compound & $k_{\mathrm{H}} / k_{\mathrm{D}}(\mathrm{AN})^{a}$ & $k_{\mathrm{H}} / k_{\mathrm{D}}(\mathrm{DCM})^{b}$ \\
\hline $\mathbf{3}$ & 0.7 & - \\
$\mathbf{4}$ & 0.9 & 0.8 \\
$\mathbf{5}$ & 0.7 & 0.75 \\
$\mathbf{6}$ & 0.8 & 0.8 \\
$\mathbf{7}$ & 0.8 & 0.75 \\
$\mathbf{8}$ & 0.8 & 0.85 \\
$\mathbf{9}$ & 0.8 & 0.7 \\
$\mathbf{1 0}$ & 0.7 & 0.95 \\
$\mathbf{1 1}$ & 0.7 & 0.9 \\
\hline
\end{tabular}

${ }^{a}$ For measurements in acetonitrile at $298 \mathrm{~K}$, rounded to nearest 0.05 . ${ }^{b}$ For measurements in dichloromethane at $298 \mathrm{~K}$, rounded to nearest 0.05 . 
forming $\mathrm{C}-\mathrm{C}$ bonds at the para position to the hydroxy or methoxy substituents. The reaction between a radical cation and its neutral precursor is a special case of this type of reaction and is often referred to as radical cationsubstrate coupling. This reaction pathway has been observed for reactions of 4,4'-dimethoxystilbene radical cation in acetonitrile. ${ }^{16}$

A particularly relevant example of the radical cationsubstrate coupling reaction is that of ethylene radical cation and the uncharged substrate, ethylene. The initial steps for the two mechanisms give the same intermediate radical cation, as illustrated in Scheme 1. Furthermore, since the term, $I_{\mathrm{N}}^{*}(\mathrm{~s})-A_{\mathrm{E}}^{*}(\mathrm{~s})$, is zero in this case, the initial gaps for both mechanisms is equal to $\Delta E_{\mathrm{ST}}\left(\pi \pi^{*}\right)$, which is expected to be of the order of $4.6 \mathrm{eV}$ $\left(106 \mathrm{kcal} \mathrm{mol}^{-1}\right) .^{9}$ In view of the known ease of the radical cation-substrate reaction for more stable radical cations, ${ }^{16,17}$ there does not appear to be reason to believe that the ethylene radical cation-ethylene reaction should have an appreciable free energy of activation.

$$
\begin{array}{r}
\left(\mathrm{CH}_{2}=\mathrm{CH}_{2}\right)^{++}+\mathrm{CH}_{2}=\mathrm{CH}_{2} \rightarrow{ }^{{ }^{\circ} \mathrm{CH}_{2} \mathrm{CH}_{2} \mathrm{CH}_{2} \mathrm{CH}_{2}{ }^{+}} \\
\text {mechanism 1 } \\
\left(\mathrm{CH}_{2}=\mathrm{CH}_{2}\right)^{\cdot+}+\mathrm{CH}_{2}=\mathrm{CH}_{2} \rightarrow{ }^{+} \mathrm{CH}_{2} \mathrm{CH}_{2} \mathrm{CH}_{2} \mathrm{CH}_{2}{ }^{\cdot} \\
\text { mechanism 2 }
\end{array}
$$

Scheme 1.

Conclusions regarding the mechanism of the reactions of $\mathrm{PAH}^{+}$with nitrogen-centered nucleophiles. The fact that there does not appear to be a positive correlation between the initial gap, formulated for either mechanism according to the CM model, and the activation barriers for the reactions of $\mathrm{PAH}^{+}$with the series of nitrogen-centered nucleophiles suggests that consideration of only the energy difference between ground state reactant configurations and a singly excited state configuration common to both reactants and products is not adequate to estimate reaction barriers in this series of reactions. Furthermore, the alternative CM model treatment (mechanism 2) presented here does not account for the trends observed in the rate constants any better than the original formulation. ${ }^{2,3}$ However, configuration 5 could contribute to the transition state for the radical cation-nucleophile reaction and decrease the 'forbiddeness' of the reaction pathway.

It is also noteworthy that the electron transfer mechanism [eqn. (2)] does not take place even in the most favorable case. This is clearly indicated by the fact that inverse secondary deuterium kinetic isotope effects, characteristic of the direct attack of the nucleophile at the 10-position of $\mathrm{PAH}^{+}$, were invariably observed. Mechanism (1) remains the most likely initial step in the radical cation-nucleophile combination reactions.

\section{Experimental}

Materials. Reagent grade acetonitrile was distilled from $\mathrm{P}_{2} \mathrm{O}_{5}$ before being passed through a column of active neutral alumina to remove water and protic impurities. Dichloromethane, after passage through active neutral alumina, was used without further purification. Tetrabutylammonium hexafluorophosphate (Aldrich) was recrystallized from dichloromethane-ether before use. 9-Phenylanthracene (Aldrich) was used as received. The nitrogen-centered nucleophiles (Aldrich) were purified by standard procedures.

Instrumentation and data handling procedures. Cyclic and linear sweep voltammetry were performed using a JAS Instrument System, J-1600-B potentiostat driven by a Hewlett-Packard 3314A function generator. After being passed through a Stanford Research Systems, Inc. model SR640 dual channel low pass filter the data were recorded on a Nicolet model 310 digital oscilloscope with 12-bit resolution. The oscilloscope and function generator were controlled by an IBM AT compatible personal computer via an IEEE interface. The current-potential curves were collected at selected trigger intervals to reduce periodic noise, ${ }^{18}$ and 20 curves were averaged before being treated with a frequency domain low pass digital filter and numerical differentiation.

Cyclic voltammetry measurements. A standard threeelectrode one-component cell was used for all kinetic measurements. Positive feedback IR compensation was used to minimize the effects of uncompensated solution resistance. Reference electrodes were $\mathrm{Ag} / \mathrm{AgNO}_{3}$ $(0.01 \mathrm{M})$ in acetonitrile constructed in the manner described by Moe. ${ }^{19}$ The working electrodes, $0.2-0.8 \mathrm{~mm} \mathrm{Pt}$, were prepared by sealing wire in glass and polishing to a planar surface as described previously. ${ }^{20}$ The working electrodes were cleaned before each series of measurements with a fine polishing powder (Struers, OP-Alumina Suspension) and wiped with a soft cloth. The cell was immersed in a water bath controlled to $25 \pm 0.2{ }^{\circ} \mathrm{C}$.

Kinetic measurements. Rate constants were obtained by comparing derivative cyclic voltammetry ${ }^{21}$ and prepeak $^{22}$ data with theoretical data obtained by digital simulation. ${ }^{23}$ The reactions were studied under secondorder conditions using solutions containing $\mathrm{PAH}$ $(1.0 \mathrm{mM})$ and nitrogen-centered nucleophile $(2.0 \mathrm{mM})$.

Acknowledgements. This research was supported by the National Science Foundation (CHE-9405656).

\section{References}

1. Parker, V. D. and Tilset, M. J. Am. Chem. Soc. 109 (1987) 2521.

2. Pross, A. J. Am. Chem. Soc. 108 (1986) 3537.

3. Shaik, S. S. and Pross, A. J. Am. Chem. Soc. 111 (1989) 4306.

4. Parker, V. D., Reitstøen, B. and Tilset, M. J. Phys. Org. Chem. 2 (1989) 580.

5. Reitstøen, B., Norrsell, F. and Parker, V. D. J. Am. Chem. Soc. 111 (1989) 8463. 
6. Reitstøen, B. and Parker, V. D. J. Am. Chem. Soc. 112 (1990) 4968.

7. Reitstøen, B. and Parker, V. D. J. Am. Chem. Soc. 113 (1991) 6954.

8. Strietwieser, A. Jr., Jagow, R. H., Fahey, R. C. and Suzuki, S. J. Am. Chem Soc. 80 (1958) 2326.

9. Aue, D. H. and Bowers, M. T. In: Bowers, M. T., Ed., Gas Phase Ion Chemistry, Academic Press, New York 1979; Vol. 2, Chap. 9.

10. McGlynn, S. P., Azumi, T. and Kinoshita, M. The Triplet State, Prentice Hall, New Jersey 1969, Chap. 3.

11. Parker, V. D., Handoo, K. L. and Reitstøen, B. J. Am. Chem. Soc. 113 (1991) 6218.

12. Kim, K., Hull, V. J. and Shine, H. J. Org. Chem. 39 (1974) 2534

13. Svanholm, U., Hammerich, O. and Parker, V. D. J. Am. Chem. Soc. 97 (1975) 101.

14. Svanholm, U. and Parker, V. D. J. Am. Chem. Soc. 98 (1976) 997.
15. Svanholm, U. and Parker, V. D. J. Am. Chem. Soc. 98 (1976) 2942.

16. Aalstad, B., Ronlan, A. and Parker, V. D. Acta Chem. Scand., Ser. B 35 (1981) 247.

17. Aalstad, B., Ronlan, A. and Parker, V. D. Acta Chem. Scand., Ser. B 35 (1981) 649; Saveant, J. M. Acta Chem. Scand., Ser. B 37 (1983) 365; Aalstad, B., Ronlan, A. and Parker, V. D. Acta Chem. Scand., Ser. B 37 (1983) 467.

18. Lasson, E. and Parker, V. D. Anal. Chem. 62 (1990) 412.

19. Moe, N. S. Anal. Chem. 46 (1974) 968.

20. Lines, R. and Parker, V. D. Acta Chem. Scand., Ser. B 31 (1977) 369.

21. Parker, V. D. Electroanal. Chem. 14 (1986) 1.

22. Parker, V. D. Acta Chem. Scand. 49 (1995).

23. Feldberg, S. W. Electroanal. Chem. 3 (1969) 199.

Received February 24, 1997. 